\title{
TODA FLOWS AND ISOSPECTRAL MANIFOLDS
}

\author{
LEONID FAYBUSOVICH
}

(Communicated by Frederick R. Cohen)

\begin{abstract}
We apply Bott's method to the calculation of Betti numbers of isospectral manifolds. Necessary properties of Toda flows, including a description of the phase portrait, are given with complete proofs.
\end{abstract}

\section{INTRODUCTION}

In the present note we show that Bott's method for the calculation of the Betti numbers of adjoint orbits of compact Lie groups [2,3] can be naturally extended to the case of more general isospectral manifolds. These manifolds appear as a generalization of the manifold of tridiagonal symmetric matrices with a fixed spectrum $[4,8]$. We use generalized Toda flows [12-14] (following mainly [8]) as an important technical tool. In particular, we describe complete phase portraits of generalized Toda flows and, as a consequence, show that these dynamical systems (and their discrete analogues, so-called QR-algorithms) possess the Morse-Smale property, i.e., are structurally stable (see in this respect also [4]). The obtained formulas for Betti numbers of isospectral manifolds are direct generalizations of those for adjoint orbits of compact Lie groups [3].

\section{Phase PORTRAit of TODa flows}

Let $(L,[]$,$) be a real Lie algebra of a semisimple noncompact Lie group G$ with a finite center. Throughout this paper we fix a Cartan involution $\theta: L \rightarrow L$ and the corresponding Cartan decomposition

$$
L=K \oplus P,
$$

where $K$ is a Lie algebra of a maximal compact subgroup $U$ of $G$. We denote as $\langle$,$\rangle the Killing form of L$ and as $($,$) the corresponding (positive definite)$ scalar product

$$
(x, y)=-\langle x, \theta y\rangle .
$$

We also fix a maximal (commutative) subalgebra $A \subset P$ and the corresponding decomposition

$$
L=L_{0} \oplus \sum_{\alpha \in \Delta} L_{\alpha}
$$

Received by the editors September 6, 1990.

1991 Mathematics Subject Classification. Primary 57N65; Secondary 58F25. 
where $\Delta$ is a restricted root system on $A$ (see [11] in connection with necessary Lie-algebraic notions). Let $C$ be a Weyl chamber in $A$ and $\Delta^{+}\left(\Delta^{-}\right)$be the set of positive (negative) roots relative to this chamber. Consider the Iwasawa decomposition of $L$

$$
L=K \oplus A \oplus\left(\sum_{\alpha \in \Delta^{+}} L_{\alpha}\right)
$$

Let $\pi: L \rightarrow L$ be the projection of $L$ onto $K$ along $A \oplus \sum\left\{L_{\alpha}: \alpha \in \Delta^{+}\right\}$. A dynamical system

$$
x=[x, \pi(x)], \quad x \in L,
$$

is called a generalized Toda flow. We denote as $\Delta_{s}^{+}$the set of simple positive roots relative to the Weyl chamber $C$. Given $\alpha \in \Delta$,

$$
\alpha=\sum_{\beta \in \Delta_{s}^{+}} n_{\beta} \beta, \quad n_{\beta} \in \mathbb{Z},
$$

we denote as $\mathrm{h}(\alpha)$ (height of $\alpha$ ) the integer

$$
\mathrm{h}(\alpha)=\sum_{\beta \in \Delta_{s}^{+}} n_{\beta} .
$$

Let

$$
V_{k}=\left(\sum\left\{L_{\alpha}: \alpha \in \Delta, \mathrm{h}(\alpha) \geq-\kappa\right\}\right)+L_{0}, \quad W_{k}=V_{k} \cap P .
$$

Proposition 1. For every $k \in \mathbb{N}$ the vector subspace $W_{k}$ is an invariant manifold for (4).

Proof. Let $\rho: L \rightarrow L$ be the projection onto $A \oplus \sum\left\{L_{\alpha}: \alpha \in \Delta^{+}\right\}$along $K$ (see (2)). Clearly $[x, \pi(x)]=[\rho(x), x]$. Further,

$$
A \subset L_{0}, \quad\left[L_{\alpha}, L_{\beta}\right] \subset L_{\alpha+\beta}, \quad \alpha, \beta \in \Delta \backslash\{0\} .
$$

By virtue of (6) we have

$$
\left[\left(A \oplus \sum_{\alpha \in \Delta^{+}} L_{\alpha}\right), V_{k}\right] \subset V_{k}, \quad k \in \mathbb{N},
$$

i.e., $[x, \pi(x)]=[\rho(x), x] \in V_{k}$, provided $x \in V_{k}$.

If $x \in P$, then $[x, \pi(x)] \in P$.Thus, $W_{k}=P \cap V_{k}$ is an invariant manifold for (4).

Remark. If we take $L=\operatorname{sl}(n, \mathbb{R}), \theta(x)=-x^{t}, k=1$, we obtain the Flaschka representation for the Toda lattice.

Lemma 1. Let $n \in C$. Then for $x \in P$

$$
\langle n,[x, \pi(x)]\rangle \geq 0,
$$

and $\langle n,[x, \pi(x)]\rangle=0$ if and only if $x \in A$.

Proof. As is easily seen (see e.g., [11])

$$
P=A \oplus\left(\sum_{\alpha \in \Delta^{+}}^{\oplus} F_{\alpha}\right),
$$


where $F_{\alpha}=\left\{x-\theta x: x \in L_{\alpha}\right\}$. Let $x \in P$. We have

$$
x=a+\sum_{\alpha \in \Delta^{+}}\left(x_{\alpha}-\theta x_{\alpha}\right)
$$

for some $a \in A, x_{\alpha} \in L_{\alpha}$. Thus,

$$
\pi(x)=-\sum_{\alpha \in \Delta^{+}}\left(x_{\alpha}+\theta x_{\alpha}\right) .
$$

We have

$$
\begin{aligned}
{[x, \pi(x)]=} & -\sum\left\{\left[a,\left(x_{\alpha}+\theta x_{\alpha}\right)\right]: \alpha \in \Delta^{+}\right\} \\
& -\sum\left\{\left[x_{\alpha}-\theta x_{\alpha}, x_{\beta}+\theta x_{\beta}\right]: \alpha \in \Delta^{+}, \beta \in \Delta^{+}\right\} .
\end{aligned}
$$

Clearly $\theta L_{\alpha}=L_{-\alpha}$ and $\left[a, x_{\alpha}+\theta x_{\alpha}\right]=\langle\alpha, a\rangle\left(x_{\alpha}-\theta x_{\alpha}\right) ;\left[x_{\alpha}-\theta x_{\alpha}, x_{\beta}+\right.$ $\left.\theta x_{\beta}\right]=\left(\left[x_{\alpha}, x_{\beta}\right]-\theta\left(\left[x_{\alpha}, x_{\beta}\right]\right)\right)+\left(\left[x_{\alpha}, \theta x_{\beta}\right]-\theta\left(\left[x_{\alpha}, \theta x_{\beta}\right]\right)\right)$. Here we made use of the following facts: $\theta([x, y])=[\theta x, \theta y], x, y \in L, \theta^{2}=I d_{L}$ (see e.g., [11]). Now $\left[x_{\alpha}, x_{\beta}\right]-\theta\left(\left[x_{\alpha}, x_{\beta}\right]\right) \in F_{\alpha+\beta},\left(\left[x_{\alpha}, \theta x_{\beta}\right]-\theta\left[x_{\alpha}, \theta x_{\beta}\right]\right) \in F_{\alpha-\beta}$ (see [11]) and consequently

$$
\begin{aligned}
\left\langle n,\left[x_{\alpha}, x_{\beta}\right]-\theta\left(\left[x_{\alpha}, x_{\beta}\right]\right)\right\rangle & =0, \quad \alpha, \beta \in \Delta^{+}, \\
\left\langle n,\left(\left[x_{\alpha}, \theta x_{\beta}\right]\right)-\theta\left(\left[x_{\alpha}, \theta x_{\beta}\right]\right)\right\rangle & =0 \quad \text { unless } \alpha=\beta .
\end{aligned}
$$

Thus, we obtain

$$
\begin{aligned}
\langle n,[x, \pi(x)]\rangle= & -\sum\left\{\langle\alpha, a\rangle\left\langle n, x_{\alpha}-\theta x_{\alpha}\right\rangle: \alpha \in \Delta^{+}\right\} \\
& -\sum\left\{\left\langle n,\left(\left[x_{\alpha}, \theta x_{\alpha}\right]-\theta\left(\left[x_{\alpha}, \theta x_{\alpha}\right]\right)\right)\right\rangle: \alpha \in \Delta^{+}\right\} .
\end{aligned}
$$

Further, $\left\langle n, x_{\alpha}-\theta x_{\alpha}\right\rangle=0, \alpha \in \Delta^{+}$and $\left\langle n,\left[x_{\alpha}, \theta x_{\alpha}\right]\right\rangle=\left\langle\left[n, x_{\alpha}\right], \theta x_{\alpha}\right\rangle=$ $\langle\alpha, n\rangle\left\langle x_{\alpha}, \theta x_{\alpha}\right\rangle=-\langle\alpha, n\rangle\left(x_{\alpha}, x_{\alpha}\right) ;\left\langle n, \theta\left(\left[x_{\alpha}, \theta x_{\alpha}\right]\right)\right\rangle=\left\langle n,\left[\theta x_{\alpha}, x_{\alpha}\right]\right\rangle=\langle[n$, $\left.\left.\theta x_{\alpha}\right], x_{\alpha}\right\rangle=-\langle\alpha, n\rangle\left\langle\theta x_{\alpha}, x_{\alpha}\right\rangle=\langle\alpha, n\rangle\left(x_{\alpha}, x_{\alpha}\right)$. Finally, we have $\langle n,[x$, $\pi(x)]\rangle=2 \sum\left\{\langle\alpha, n\rangle\left(x_{\alpha}, x_{\alpha}\right): \alpha \in \Delta^{+}\right\}$, i.e., $\langle n,[x, \pi(x)]\rangle \geq 0$ and $\langle n$, $[x, \pi(x)]\rangle=0$ if and only if $\left(x_{\alpha}, x_{\alpha}\right)=0, \alpha \in \Delta^{+}$. In other words, $(\langle n,[x, \pi(x)]\rangle=0) \Leftrightarrow\left(x_{\alpha}=0, \alpha \in \Delta^{+}\right) \Leftrightarrow x \in A$.

Given $p \in P$, denote as $O(p) \subset P$ the orbit of $p$ under the adjoint action of $U$ on $P$. Since $U$ is a compact Lie group, every orbit $O(p)$ is a smooth manifold. Denote as $T_{q}(O(p))$ the tangent space to $O(p)$ at a point $q \in O(p)$. Clearly $T_{q}(O(p))=\{[q, \xi]: \xi \in K\}$. This implies

Lemma 2. Every orbit $O(p)$ is an invariant manifold for the dynamical system (4).

From Lemmas 1 and 2 it follows that

Corollary 1. The set of the equilibrium points of the dynamical system (4) on $O(p)$ coincides with the set $A \cap O(p)$.

As is known (see, e.g., [11]), for every $p \in P$ the set $A \cap O(p)$ is nonempty, finite, and coincides with an orbit of the Weyl group $W$, i.e., $A \cap O(p)=W q$, where $q$ is a unique element of $\overline{\mathrm{C}} \cap O(p)$ (we denote as $\overline{\mathrm{C}}$ the closure of the Weyl chamber $C$ ).

We need the following simple Lemma. 
Lemma 3. Given $p \in P$, let $O(p) \cap A=\left\{a_{1}, \ldots, a_{k}\right\}$ for some $k \in \mathbb{N}$. There always exists $n \in C$ such that $\left\langle a_{i}, n\right\rangle \neq\left\langle a_{j}, n\right\rangle$, provided $i \neq j$.

Proposition 2. Let $x(t)$ be a solution to (4) such that $x(0)=p$. Then $x(t) \rightarrow$ $p_{ \pm}, t \rightarrow \pm \infty$, for some $p_{ \pm} \in A \cap O(p)$.

Proof. By Lemma 3 there is $n \in C$ such that $\langle n, a\rangle \neq\langle n, b\rangle$ for every $a, b \in$ $A \cap O(p), a \neq b$. The function $f(x)=\langle x, n\rangle$ is a Lyapunov function for (4) on $O(p)$ by Lemma 1 . The result follows now by a standard reasoning.

Remark. Proposition 2 is well known (compare with [10]). But our proof seems to be new. We will use the Lyapunov function $x \rightarrow\langle x, n\rangle$ as a main technical tool throughout this paper.

Let $M\left(M^{*}\right)$ be the stabilizer (normalizer) of $A$ in $U$. Then the Weyl group $W$ of $L$ (relative to $\theta, A$ ) is isomorphic with $M^{*} / M$ (see [11]). Let $G_{A}$ and $N$ be connected Lie subgroups of $G$ with Lie algebras $A$ and $\sum\left\{L_{\alpha}: \alpha \in \Delta^{+}\right\}$, respectively. We denote as $B$ the Borel subgroup $M G_{A} N$. As is known (see [11]) $B$ is a closed subgroup of $G$. Let $G / B$ to the corresponding coset space and $\mu: G \rightarrow G / B$ be a canonical projection. The group $G$ acts from the left on $G / B$. Given $p \in C$, let $\varphi: O(p) \rightarrow G / B$ be the mapping such that $\varphi(\operatorname{Ad}(g) p)=g \cdot o$, where $g \in U, o=\mu(B)$. The mapping $\varphi$ defines a smooth isomorphism of $O(p)$ with $G / B$ ([11]).

Let $w \in M^{*} / M \approx W$ and $w=m^{*} \cdot M$ for some $m^{*} \in M^{*}$. Then $w \cdot o=$ $m^{*} \cdot o$ does not depend on the choice of a representative $m^{*} \in M^{*}$ because $M \subset B$ and $M \cdot o=o$. Recall that [11] the sets $B \cdot(w \cdot o), w \in W$ are pairwise disjoint and, moreover,

$$
G / B=\bigcup_{w \in W} B \cdot(w \cdot o),
$$

(Bruhat decomposition of $G / B$ ). These sets (which, in fact, are submanifolds of $G / B)$ are called the Bruhat cells of this decomposition. We obtain a Bruhat decomposition of $O(p), p \in C$, with the help of the isomorphism $\varphi$ :

$$
O(p)=\bigcup_{w \in W} \varphi^{-1}(B \cdot w \cdot o) .
$$

Proposition 3. The Bruhat cells (9) are invariant manifolds for the dynamical system (4).

Proof. Let $x_{0} \in O(p), p \in C$ and $\exp \left(x_{0} t\right)=Q(t) R(t)$, where $t \in \mathbb{R}, Q(t) \in$ $U, R(t) \in G_{A} N$. There is such a unique decomposition (Iwasawa decomposition, see [11]). Then $x(t)=\operatorname{Ad}\left(Q(t)^{-1}\right) \cdot x_{0}$ is the solution to (4) such that $x(o)=x_{0}$ (for a proof see [10]). There is $Q_{0} \in U$ such that $\operatorname{Ad}\left(Q_{0}^{-1}\right) \cdot p=x_{0}$. We have $\varphi(x(t))=Q(t)^{-1} \cdot Q_{0}^{-1} \cdot o=R(t) \cdot \exp \left(-x_{0} t\right) \cdot Q_{0}^{-1} \cdot o=R(t) \cdot Q_{0}^{-1}$. $\exp \left(-t \operatorname{Ad}\left(Q_{0}\right) x_{0}\right) \cdot o=R(t) \cdot Q_{0}^{-1} \cdot \exp (-p t) \cdot o=R(t) \cdot Q_{0}^{-1} \cdot o=R(t) \cdot \varphi\left(x_{0}\right)$. Here we used that $\exp (-p t) \cdot o=o, p \in A$. Now $R(t) \in G_{A} N \subset B$. Whence $\varphi(x(t))$ belongs to the $B$-orbit of $\varphi\left(x_{0}\right)$.

Let $w \in W \approx M^{*} / M, w=m^{*} \cdot M$ for some $m^{*} \in M^{*}$. Then the Lie subgroup $m^{*} \cdot B \cdot\left(m^{*}\right)^{-1}$ does not depend on the choice of a representative $m^{*} \in M^{*}$ because $M$ normalizes $B$. Thus, we obtain the Lie subgroup 
$B_{w}=m^{*} \cdot B \cdot\left(m^{*}\right)^{-1}$. Given $w_{1} \in W$, we obtain from (8) the following decomposition

$$
G / B=\bigcup_{w \in W} B_{w_{1}} \cdot(w \cdot o)
$$

There is a unique element $w_{m} \in W$ such that $w_{m} \cdot \Delta^{+}=\Delta^{-}$(see, e.g., [11]). In particular, the following Bruhat decomposition occurs:

$$
G / B=\bigcup_{w \in W} B_{w_{m}} \cdot(w \cdot o)
$$

On the other hand, let $\Theta$ be the automorphism of $G$ with $T_{e}(\Theta)=\theta$. Clearly $\boldsymbol{\Theta} \cdot \boldsymbol{B}=B_{u}, u=w_{m}$. If $\exp \left(x_{0} t\right)=Q(t) \cdot R(t), Q(t) \in U, R(t) \in G_{A} N, x_{0} \in$ $P$, and $t \in \mathbb{R}$, then $\exp \left(\theta\left(x_{0}\right) t\right)=Q(t)[\Theta R(t)]$, or $Q(t)^{-1}=[\Theta R(t)] \cdot \exp \left(x_{0} t\right)$. As in the case of proposition 3, we have that $\varphi(x(t))$ belongs to the $B_{u}$-orbit of $\varphi\left(x_{0}\right)$, where $u=w_{m}$. Thus, we proved:

Proposition 4. The Bruhat cells of the decomposition (10) (i.e., $B_{u}$-orbits in $G / B, u=w_{m}$ ) are invariant manifolds for (4).

Given $n \in C$, consider a mapping $F: \mathbb{R} \times G / B \rightarrow G / B, F(t, z)=\exp (t n) \cdot z$. Clearly, $F\left(t_{2}, F\left(t_{1}, z\right)\right)=F\left(t_{2}+t_{1}, z\right), t_{1}, t_{2} \in \mathbb{R}$. Thus, there exists a vector field $X_{n}$ on $G / B$ such that $X_{n}(z)=T_{0}\left(F_{z}\right) \cdot 1, F_{z}(t)=\exp (t n) \cdot z, t \in \mathbb{R}$ and $z \in G / B$. If $w \in W, w=m^{*} \cdot M, m^{*} \in M^{*}$, then $\exp (t n) \cdot(w \cdot o)=$ $m^{*} \exp \left(t \operatorname{Ad}\left(m^{*}\right)^{-1} \cdot n\right) \cdot o$. But $\operatorname{Ad}\left(m^{*}\right)^{-1} \cdot n \in A$. Whence, $\exp (t n) \cdot(w \cdot o)=$ $w \cdot o$.

Proposition 5. The Bruhat cell $B \cdot(w \cdot o)$ (respectively, $B_{u} \cdot(w \cdot o), u=w_{m}$ ) is the unstable (respectively, stable) manifold of the equilibrium point $w \cdot o$ of the vector field $X_{n}$.

Proof. We have $B=M \cdot G_{A} \cdot N$ and every element $y \in N$ has the form $y=$ $\exp \left(\sum\left\{x_{\alpha}: \alpha \in \Delta^{+}\right\}\right), a_{\alpha} \in L_{\alpha}$ (see [11]). Let $b=c \cdot y$, where $c \in M G_{A}$. We have

$$
\begin{aligned}
& \exp (n t) \cdot b \cdot(w \cdot o) \\
& \quad=\exp (n t) \cdot c \cdot \exp (-n t) \cdot \exp \left(\sum\left\{\exp \operatorname{ad}(n t) \cdot x_{\alpha}: \alpha \in \Delta^{+}\right\}\right) \cdot \exp (n t) \cdot w \cdot o .
\end{aligned}
$$

But $\exp (n t) \cdot c \cdot \exp (-n t)=c$ because $G_{A}$ is a commutative group, $\exp (n t) \in$ $G_{A}$ and $M$ stabilizes $G_{A}$. Further, $\operatorname{ad}(n t) x_{\alpha}=t\langle\alpha, n\rangle x_{\alpha}$ and consequently $\exp (t \operatorname{ad} n) \cdot x_{\alpha}=\exp (t\langle\alpha, n\rangle) \cdot x_{\alpha} \rightarrow 0, t \rightarrow-\infty$ because $\langle\alpha, n\rangle>0, \alpha \in \Delta^{+}$. This implies $\exp (n t) \cdot b \cdot w \cdot o \rightarrow w \cdot o$. Similarly $\exp (n t) \cdot b_{m} \cdot w \cdot o \rightarrow w \cdot o$, $t \rightarrow+\infty$, where $b_{m} \in B_{u}, u=w_{m}$.

The isomorphism $\varphi: O(p) \rightarrow G / B$ enables us to carry over the vector field $X_{n}$ to the $O(p)$. We denote this vector field as $Y_{n}$.

Proposition 6. Given $n \in C$, the following holds:

$$
\left\langle n, Y_{n}(x)\right\rangle \geq 0, \text { for every } x \in O(p) .
$$

Proof. Let $x \in O(p)$ and $\varphi(x)=Q \cdot o, Q \in U$. Consider the Iwasawa decomposition

$$
\exp (t n) \cdot Q=Q(t) \cdot R(t)
$$


where $t \in \mathbb{R}, Q(t) \in U, R(t) \in G_{A} N$. The integral curve $x(t)$ of the vector field $Y_{n}$ such that $x(0)=x$ takes the form: $x(t)=\varphi^{-1}(\exp (n t) \cdot \varphi(x))=$ Ad $Q(t) \cdot p$. Differentiating (11) with respect to $t$, we obtain $\operatorname{Ad}\left(Q(t)^{-1}\right) \cdot n=$ $Q(t)^{-1} \cdot d Q(t) / d t+d R(t) / d t \cdot R(t)^{-1}$. Thus, we have

$$
Q(t)^{-1} \cdot d Q(t) / d t=\pi\left(\operatorname{Ad}\left(Q(t)^{-1}\right) \cdot n\right) .
$$

This implies that

$$
\begin{aligned}
d / d t(\langle n, x(t)\rangle) & =d / d t(\langle n, \operatorname{Ad}(Q(t)) \cdot p\rangle)=d / d t\left(\left\langle\operatorname{Ad}\left(Q(t)^{-1}\right) \cdot n, p\right\rangle\right) \\
& =\left(\left\langle\left[\operatorname{Ad}\left(Q(t)^{-1}\right) \cdot n, \pi\left(\operatorname{Ad}\left(Q(t)^{-1}\right) \cdot n\right], p\right\rangle\right) .\right.
\end{aligned}
$$

This yields

$$
\left\langle n, Y_{n}(x)\right\rangle=\left\langle\left[\operatorname{Ad}\left(Q^{-1}\right) \cdot n, \pi\left(\operatorname{Ad}\left(Q^{-1}\right)\right) \cdot n\right], p\right\rangle \geq 0
$$

by Lemma 1 .

Proposition 7. Every equilibrium point $w \cdot p, w \in W$, of the dynamical system (4) on the orbit $O(p), p \in C$, is hyperbolic and

$$
\operatorname{ind}(w \cdot p)=\sum\left\{\operatorname{dim} L_{\alpha}: \alpha \in \Delta^{+}, w^{-1}(\alpha) \in \Delta^{+}\right\} .
$$

Proof. Denote as $A_{w}: T_{w p}(O(p)) \rightarrow T_{w p}(O(p))$ the linearization of the dynamical system (4) at the equilibrium point $w \cdot p$. Clearly $A_{w}$ is the restriction of the linear operator $\xi \rightarrow[w p, \pi(\xi)]: P \rightarrow P$ to $T_{w p}$. We have $T_{w p}(O(p))=\sum_{\alpha \in \Delta^{+}} F_{\alpha}$. Let $\left(\xi_{\alpha}-\theta \xi_{\alpha}\right) \in F_{\alpha}, \xi_{\alpha} \in L_{\alpha}$. Then $A_{w}\left(\xi_{\alpha}-\theta \xi_{\alpha}\right)=$ $\left[w p, \pi\left(\xi_{\alpha}-\theta \xi_{\alpha}\right)\right]=-\left[w p, \xi_{\alpha}+\theta \xi_{\alpha}\right]=-\langle\alpha, w p\rangle\left(\xi_{\alpha}-\theta \xi_{\alpha}\right)$. Thus, ind $A_{w}=$ $\sum\left\{\operatorname{dim} L_{\alpha}: \alpha \in \Delta^{+}, w^{-1}(\alpha) \in \Delta^{+}\right\}$.

Theorem 1. The Bruhat cell $\varphi(B \cdot w \cdot o)$ (respectively, $\varphi\left(B_{u} \cdot w \cdot o\right), u=w_{m}$ ) is the unstable (respectively, stable) manifold for the equilibrium point $w \cdot p$ of the dynamical system (4) on the orbit $O(p), p \in C$.

Remark. For the notations used see (10).

Proof. By Lemma 3 there is $n \in C$ such that $\left\langle n, w_{2} \cdot p\right\rangle \neq\left\langle n, w_{1} \cdot p\right\rangle$ if $w_{1} \neq$ $w_{2}$. Denote as $f_{n}$ the function $x \rightarrow\langle n, x\rangle: P \rightarrow \mathbb{R}$. Let $\varepsilon>0$ be such that $\varepsilon<\min \left\{\left|f_{n}\left(w_{2} p\right)-f_{n}\left(w_{1} p\right)\right|: w_{2} \neq w_{1} \in W\right\}$. Let us choose an open neighbourhood $V_{\varepsilon}(w p)$ of the point $w p$ in $P$ such that $\left|f_{n}(x)-f_{n}(w p)\right|<$ $\varepsilon, x \in V_{\varepsilon}(w p)$. Denote as $C(w)$ the cell $\varphi\left(B_{u} \cdot w \cdot o\right), u=w_{m}$, and as $F: \mathbb{R} \times O(p) \rightarrow O(p), F_{n}: \mathbb{R} \times O(p) \rightarrow O(p)$ the flows of the vector fields (4) and $Y_{n}$, respectively. Let $x \in C(w) \cap V_{\varepsilon}(w p)$. By Proposition $2 \lim F(t, x)=$ $w_{1} \cdot p, t \rightarrow+\infty$, for some $w_{1} \in W$. By Lemma $1, f_{n}\left(w_{1} p\right) \geq f_{n}(x)$. But $f_{n}(x)>f_{n}(w p)-\varepsilon$ because $x \in V_{\varepsilon}(w p)$. Thus, $f_{n}\left(w_{1} \cdot p\right)>f_{n}(w p)-\varepsilon$. This implies (due to the choice of $\varepsilon$ ) that $f_{n}\left(w_{1} \cdot p\right) \geq f_{n}(w p)$. Clearly there is $T>0$ such that $\left|f_{n}(F(t, x))-f_{n}\left(w_{1} \cdot p\right)\right|<\varepsilon$ if $t \geq T$. By Proposition 4, $F(t, x) \in C(w), t \in \mathbb{R}$ and consequently (Proposition 5) $\lim F_{n}\left(t, F\left(t^{*}, x\right)\right)=$ $w p, t \rightarrow+\infty$ for every $t^{*} \in \mathbb{R}$. By Proposition $6, f_{n}(w p) \geq f_{n}\left(F\left(t^{*}, x\right)\right)$ for every $t^{*} \in \mathbb{R}$. In particular, $f_{n}(w p) \geq f_{n}(F(T, x))>f_{n}\left(w_{1} \cdot p\right)-\varepsilon$, i.e., $f_{n}(w p) \geq f_{n}\left(w_{1} \cdot p\right)$. Thus, $f_{n}(w p)=f_{n}\left(w_{1} \cdot p\right)$ and hence $w p=w_{1} \cdot p$ by the choice of $n$. As is known $B_{u} \cdot w \cdot o$ is a smooth submanifold of $G / B$ and $\operatorname{dim}\left(B_{u} \cdot w \cdot o\right)=\sum\left\{\operatorname{dim} L_{\alpha}: \alpha>0, w^{-1}(\alpha)>0\right\}$ (see, e.g., [11]). Here $u=w_{n}$. Thus, $\operatorname{dim} C(w)=\operatorname{ind}(w p)$ by Proposition 7. If we denote as 
$S(w p)$ the stable manifold of (4) on the $O(p)$, then, what we have proven means that there is a neighbourhood $V^{\prime}(w p)$ of the point $w p$ in $P$ such that $V^{\prime}(w p) \cap S(w p)=V^{\prime}(w p) \cap C(w)$. This holds for every $w \in W$. If $x \in C(w)$ and $F(t, x) \rightarrow w_{1} \cdot p, t \rightarrow+\infty$, then for sufficiently large $t$, $F(t, x) \in V^{\prime}\left(w_{1} \cdot p\right) \cap S\left(w_{1} \cdot p\right)$ and hence $F(t, x) \in C\left(w_{1}\right)$. But by virtue of Proposition 4, $F(t, x) \in C(w)$. Thus, $C\left(w_{1}\right) \cap C(w) \neq \varnothing$, i.e., $C\left(w_{1}\right)=C(w)$ and $w_{1}=w$. This proves that $S(w p)=C(w), w \in W$. The proof for the case of unstable manifolds is similar (but relies upon Proposition 3 instead of Proposition 4).

Remark. The idea of this proof is due to Atiyah and Bott [1, Proposition 1.19].

Corollary. The Toda flow (4) on the orbit $O(p), p \in C$, is a Morse-Smale vector field.

Proof. We must show that stable and unstable manifolds of (4) intersect each other transversally. Let $x \in S(w p) \cap U\left(w_{1} \cdot p\right)$, where we denote as $U(w p)$ the unstable manifold of $w p, w \in W$. Then $S(w p)=B_{u} \cdot x, u=w_{m}$ and $U\left(w_{1} \cdot p\right)=B \cdot x$ by Theorem 1 . But

$$
L(B) \oplus L\left(B_{w_{m}}\right)=A \oplus M \oplus\left(\sum_{\alpha>0} L_{\alpha}\right) \oplus\left(\sum_{\alpha<0} L_{\alpha}\right)=L .
$$

Thus, $T_{x}(S(w p)) \oplus T_{x}\left(U\left(w_{1} \cdot p\right)\right)=T_{x}(O(p))$.

Remark. The so-called $Q R$-algorithm associated with (4) can be described as a discrete dynamical system on $\Pi=\exp (P)$ as follows. If $F: \mathbb{R} \times P \rightarrow P$ is the flow of (4) and $G: \mathbb{Z} \times \Pi \rightarrow \Pi$ is the flow of the associated $Q R$-algorithm, then

$$
G(k, \exp x)=\exp (F(k, x)), \quad k \in \mathbb{Z}, \quad x \in P,
$$

(see, e.g., [4, 10]). This implies that $Q R$-algorithm, associated with (4) is a Morse-Smale dynamical system on the every orbit $\operatorname{Or}(p)=\exp [O(p)]$. This is clear because exp maps $P$ diffeomorphically onto $\Pi$.

Remark. Theorem 1 along with Corollary for the case $L=\operatorname{sl}(n, \mathbb{R})$ was proved in [6] with the help of a different technique [5, 7].

\section{ISOSPECTRAL MANIFOLDS}

The mapping $\psi: U / M \times C \rightarrow P, \psi(g, p)=\operatorname{Ad}(g) p$ is a diffeomorphism onto an open subset $P^{\prime}$ in $P$ (see, e.g., [11]). Thus, the mapping $\nu: P^{\prime} \rightarrow C$, $\nu(p)=\pi_{2} \cdot \psi^{-1}(p), \pi_{2}: U \times C \rightarrow C, \pi_{2}(g, p)=p$ is a submersion. Clearly $O(p)=\nu^{-1}(\nu(p))$. The set $W_{k}^{\prime}=W_{k} \cap P^{\prime}$ is an open submanifold of $W_{k}$, $k \geq 1$. Denote as $\nu_{k}$ the restriction of $\nu$ on $W_{k}^{\prime}$.

Proposition 8. Given $p \in C$, the mapping $T_{x}\left(\nu_{k}\right): T_{x}\left(W_{k}^{\prime}\right) \rightarrow T_{x}(C)$ is a submersion at every point $x \in O(p) \cap W_{k}^{\prime}=\nu_{\kappa}^{-1}\left(\nu_{k}(p)\right)=I_{k}$. The set $I_{k}$ is a smooth compact orientable manifold; $\operatorname{dim} I_{k}=\operatorname{dim} W_{k}-\operatorname{dim} A, k \geq 1$.

Proof. Let $A^{\prime}=\{a \in A:\langle\alpha, a\rangle \neq 0, \forall \alpha \in \Delta\}$. Consider the mapping $i_{k}: A^{\prime} \rightarrow W_{k}^{\prime}, i_{k}(a)=a$. As is known, $A^{\prime}=\bigcup\{w \cdot C: w \in W\}$ (see, e.g., [11]). If $a \in w \cdot C$, then $\nu_{k} \cdot i_{k}(a)=w^{-1} a$. This implies that $T_{a}\left(\nu_{k} \cdot i_{k}\right)=$ $T_{i k(a)}\left(\nu_{k}\right) \cdot T_{a}\left(i_{k}\right): A \rightarrow A$ coincides with the mapping $b \rightarrow w^{-1} b, b \in A$. Consequently $\operatorname{Im} T_{a}\left(\nu_{k}\right)=A$, i.e., $\nu_{k}$ is a submersion at every point $a \in A^{\prime}$. 
Let $F: \mathbb{R} \times P \rightarrow P$ be the flow of the dynamical system (4) and $x \in O(p) \cap W_{k}^{\prime}$. By Proposition 2, $F(x, t) \rightarrow w p, t \rightarrow+\infty$, for some $w \in W$. Denote as $F_{t}: P \rightarrow P$ the mapping $F_{t}(y)=F(t, y), t \in \mathbb{R}$. By Proposition $1, T_{y}\left(F_{t}\right)$ maps $T_{y}\left(W_{k}\right)=W_{k}$ isomorphically onto $T_{F(t, y)}\left(W_{k}\right)=W_{k}$. By Lemma 2, $\nu_{\kappa}\left(F_{t}(x)\right)=\nu_{k}(x)$. Hence

$$
T_{F_{t}(y)}\left(\nu_{k}\right) \circ T_{x}\left(F_{t}\right)=T_{x}\left(\nu_{k}\right), \quad x \in O(p) .
$$

For sufficiently large $t, T_{F(t, x)}\left(\nu_{k}\right)$ is a surjection because $T_{w p}\left(\nu_{k}\right)$ is a surjection and $F_{t}(x) \rightarrow w p, t \rightarrow+\infty$. By (11) we obtain that $T_{x}\left(\nu_{k}\right)$ is also a surjection. Thus, we have proven that $\nu_{k}$ is a submersion at every point $x \in I_{k}$. This implies that $I_{k}=\nu_{k}^{-1}\left(\nu_{k}(p)\right)$ is an orientable manifold; $\operatorname{dim} I_{k}=$ $\operatorname{dim} W_{k}-\operatorname{dim} A$.

Let us observe that $O(p)$ and $W_{k}$ intersect each other transversally at the points $w p, w \in W$. In particular $T_{w p}\left(I_{k}\right)=T_{w p}(O(p)) \cap W_{k}$, i.e.,

$$
T_{w p}\left(I_{k}\right)=\sum_{\substack{\alpha \in \Delta^{+} \\ h(\alpha) \leq k}} F_{\alpha} .
$$

Remark. The idea of this proof is due to Fried [8], who considered the case $L=\operatorname{sl}(n, \mathbb{R})$.

Corollary. For every $k \geq 1, I_{k}$ is a connected manifold.

Proof. Let $Y_{k}$ be the restriction of the Toda vector field (4) to $I_{k}$. As in the proof of Proposition 7 (taking into account (12)) we show that the index of $Y_{k}$ at $w p$, which we denote as $\operatorname{ind}_{k}(w p)$ takes the form

$$
\operatorname{ind}_{k}(w p)=\sum\left\{\operatorname{dim} L_{\alpha} ; \alpha \in \Delta^{+}, w^{-1}(\alpha) \in \Delta^{+}, h(\alpha) \leq k\right\} .
$$

In particular, there is exactly one stable equilibrium point $p$ and $\operatorname{ind}_{k}(p)=$ $\operatorname{dim} I_{k}$. Indeed, if $w \neq e$, then there is $\alpha \in \Delta_{s}^{+}$(a simple positive root relative to $C$ ) such that $w^{-1}(\alpha)<0$ (see, e.g., [11]). But $\left(\alpha \in \Delta_{s}^{+}\right) \Rightarrow(h(\alpha)=1 \leq k)$. Clearly the stable manifold of $p$ on $I_{k}$ is an open, dense, and connected set in $I_{k}$. In particular, $I_{k}$ is also a connected set.

Let $p \in C$ and $f$ be a smooth function, defined on some open neighbourhood of the orbit $O(p)$. Consider the problem

$$
f(q) \rightarrow \min , \quad q \in O(p) .
$$

Proposition 9. The stationary points of the problem (13) are exactly the points $q_{0} \in O(p)$ such that

$$
\left[\nabla f\left(q_{0}\right), q_{0}\right]=0 .
$$

Here $\nabla f(q) \in P$ is by definition such that $\langle\nabla f(q), \xi\rangle=d f(q) \cdot \xi, \xi \in P$. If $q_{0}$ is a stationary point of (13), then the Hessian $H\left(q_{0}\right)$ at this point takes the form

$$
\begin{aligned}
H\left(q_{0}\right)\left(\left[\xi, q_{0}\right],\left[\xi, q_{0}\right]\right)= & D^{2} f\left(q_{0}\right) \cdot\left(\left[\xi, q_{0}\right],\left[\xi, q_{0}\right]\right) \\
& +\left\langle\nabla f\left(q_{0}\right),\left[\xi,\left[\xi, q_{0}\right]\right]\right\rangle, \quad \xi \in K .
\end{aligned}
$$

Proof. Clearly $T_{q}(O(p))=[\xi, q], \xi \in \cdot K$. Thus, $(d f(q)([\xi, q])=0, \forall \xi \in$ $K) \Leftrightarrow(\langle\nabla f(q),[\xi, q]\rangle=0, \forall \xi \in K) \Leftrightarrow([\nabla f(q), q]=0)$. If $\mu: N_{1} \rightarrow N_{2}$ is a submersion of manifolds $N_{i}, i=1,2$ and $r: N_{2} \rightarrow \mathbb{R}$ is a smooth function, 
then, given a critical point $z$ of $r$, every $z^{\prime} \in \mu^{-1}(z)$ is a critical point of $r \cdot \mu$. Moreover, if $H_{z}$ and $H_{z^{\prime}}$ are Hessians of $r$ and $r \cdot \mu$ at the points $z$ and $z^{\prime}$, respectively, then $H_{z}\left(T_{z^{\prime}}(\mu) \cdot \xi, T_{z^{\prime}}(\mu) \cdot \xi\right)=H_{z^{\prime}}(\xi, \xi), \xi \in T_{z^{\prime}}\left(N_{2}\right)$. This is verified by a direct calculation in local coordinates.

If $q_{0}$ is a critical point of $f$, then the mapping $\xi \rightarrow \exp (\operatorname{ad} \xi) \cdot q_{0}$ is a submersion of a neighbourhood of zero in $K$ onto some neighbourhood of $q_{0}$ in $O(p)$. Consider the function $\varphi(\xi)=f\left(\exp (\operatorname{ad} \xi) \cdot q_{0}\right), \xi \in K$. We have $\varphi(\xi)=f\left(q_{0}+\left[\xi, q_{0}\right]+1 / 2\left(\left[\xi,\left[\xi, q_{0}\right]\right]\right)+o\left(\|\xi\|^{2}\right)\right)=f\left(q_{0}\right)+D f\left(q_{0}\right) \cdot\left(\left[\xi, q_{0}\right]+\right.$ $\left.1 / 2\left(\left[\xi,\left[\xi, q_{0}\right]\right]\right)\right)+1 / 2\left(D^{2} f\left(q_{0}\right)\left(\left[\xi, q_{0}\right],\left[\xi, q_{0}\right]\right)\right)+o\left(\|\xi\|^{2}\right)$. Hence $D^{2} \varphi(0)(\xi, \xi)$ $=D f\left(q_{0}\right) \cdot\left[\xi,\left[\xi, q_{0}\right]\right]+D^{2} f\left(q_{0}\right)\left(\left[\xi, q_{0}\right],\left[\xi, q_{0}\right]\right)$. But $H\left(q_{0}\right)\left(\left[\xi, q_{0}\right],\left[\xi, q_{0}\right]\right)=$ $D^{2} \varphi(0)(\xi, \xi)$.

Consider the special case of the problem (13): $f(q)=f_{n}(q)=\langle n, q\rangle, n \in$ $C$. By Proposition 9 the stationary points are determined by the condition $[q, n]=0, q \in O(p)$, i.e., the set of stationary points coincides with $A \cap O(p)=$ $W \cdot p$. Our ultimate goal is the following problem

$$
\langle n, q\rangle \rightarrow \min , \quad q \in\left(O(p) \cap W_{k}\right)=I_{k}, \quad k \geq 1 .
$$

Proposition 10. The set of stationary points for the problem (16) is $A \cap O(p)=$ $W \cdot p$. The Hessian $H_{k}(w p)$ at the point $w p, w \in W$ takes the form

$$
H_{k}(w p)([\xi, w p],[\xi, w p])=-\sum_{\substack{\alpha>0 \\ h(\alpha) \leq k}}\left\langle w^{-1} \alpha, p\right\rangle\langle\alpha, n\rangle\left(\xi_{\alpha}-\theta \xi_{\alpha}, \xi_{\alpha}-\theta \xi_{\alpha}\right)
$$

where $\xi=\sum\left\{\left(\xi_{\alpha}+\theta \xi_{\alpha}\right): \alpha>0, h(\alpha) \leq k\right\}, \xi_{\alpha} \in L_{\alpha}$. In particular, every critical point is nondegenerate and

$$
\text { ind } H_{k}(w p)=\sum_{\substack{\alpha>0 \\ h(\alpha) \leq k \\ w^{-1}(\alpha)>0}}\left(\operatorname{dim} L_{\alpha}\right) .
$$

Proof. Since $I_{k}$ is a submanifold of $O(P)$, every point from $A \cap O(p)$ is also a stationary point of $I_{k}$. Let $x \in I_{\kappa}, x \notin A \cap O(p)$. Then by Proposition 1 and Lemma 2, $[x, \pi(x)] \in T_{x}\left(I_{k}\right)$. But $d f(x)([x, \pi(x)])=\langle n,[x, \pi(x)]\rangle>0$ by Lemma 1 . Hence, $x$ is not a stationary point for $f$ on $I_{k}$. The explicit expression for the Hessian $H_{k}(w p)$ follows from (12) and (15) by a direct calculation.

Let $L$ be the Lie algebra of a complex semisimple Lie group. We consider $L$ as a Lie algebra over $\mathbb{R}$. But $L$ is endowed with a complex structure. Let $\left(h_{\alpha}, e_{\alpha}, e_{-\alpha}\right), \alpha \in \Delta^{+}$, be a Chevalley basis for $L$, i.e., $h_{\alpha}=\left[e_{\alpha}, e_{-\alpha}\right]$, $\alpha \in \Delta^{+},\left[e_{\alpha}, e_{\beta}\right]=N_{\alpha, \beta} \cdot e_{\alpha+\beta}$, where $N_{\alpha, \beta} \in \mathbb{R}, N_{\alpha, \beta}=0$ if $\alpha+\beta \notin$ $\Delta \cup\{0\}, N_{-\alpha,-\beta}=-N_{\alpha, \beta}, \alpha, \beta \in \Delta$. Let, further, $H=\operatorname{span}_{\mathbb{C}}\left(h_{\alpha}, \alpha \in \Delta^{+}\right)$, $H_{\mathbb{R}}=\{h \in H: \alpha(h) \in \mathbb{R}, \forall \alpha \in \Delta\}$. Set $f_{\alpha}=e_{\alpha}-e_{-\alpha}, g_{\alpha}=i\left(e_{\alpha}+e_{-\alpha}\right)$, $\alpha \in \Delta^{+} ; K=\operatorname{span}_{\mathbb{R}}\left(f_{\alpha}, g_{\alpha}, \alpha \in \Delta^{+}\right) \oplus i H_{\mathbb{R}}, A=H_{\mathbb{R}}, L_{\alpha}=\mathbb{C} e_{\alpha}, \alpha \in \Delta$, $P=\operatorname{span}_{\mathbb{R}}\left(i f_{\alpha}, i g_{\alpha}\right) \oplus H_{\mathbb{R}}$. Clearly $L=K \oplus P$ is a Cartan decomposition and $\operatorname{dim}_{\mathbb{R}} L_{\alpha}=2, \alpha \in \Delta$.

Theorem 2. In the situation described above (i.e., for the case that $L$ is the Lie algebra of a complex semisimple Lie group) the Betti numbers $\beta_{s}\left(I_{k}\right), k \geq 1$, $0 \leq s \leq \operatorname{dim} I_{k}$ take the form

$$
\beta_{2 l+1}\left(I_{k}\right)=0, \quad \beta_{2 l}\left(I_{k}\right)=\operatorname{card}\left\{w \in W: \gamma_{\kappa}(w)=l\right\},
$$


where $\gamma_{k}(w)=\operatorname{card}\left\{\alpha>0: w^{-1}(\alpha)>0, h(\alpha) \leq k\right\}$. Besides, $I_{k}$ are simply connected manifolds.

Proof. By Proposition 10 in this situation all indices ind $H_{k}(w p)$ are even. Hence $f_{n}$ is a perfect Morse function on $I_{k}$ (see, e.g., [1]). The expression for Betti numbers follows from (18). From the proof of Corollary of Proposition 7 we know that the restriction of the Toda vector field to $I_{k}$ has exactly one stable equilibrium point whose stable manifold is open, dense in $I_{k}$ and is clearly simply connected. In the situation considered stable manifolds of other equilibrium points have codimensional greater or equal to 2 . Thus, $I_{k}$ is itself simply connected.

Remark. The Betti numbers (19) coincide with those of a so-called Hessenberg varieties (see $[9,15])$. This is not accidental because isospectral manifolds $I_{k}$ and corresponding Hessenberg varieties coincide modulo action of a toral group.

Remark. If $L$ is the Lie algebra of a complex semisimple Lie group as above, we can consider the real split form $L_{s}$ of $L, L_{s}=\left(\sum_{\alpha \in \Delta} \mathbb{R} e_{\alpha}\right) \oplus H_{\mathbb{R}}$. If we take

$$
K_{s}=\sum_{\alpha \in \Delta^{+}} \mathbb{R}\left(e_{\alpha}-e_{-\alpha}\right), \quad P_{s}=H_{\mathbb{R}} \oplus \sum_{\alpha \in \Delta^{+}} \mathbb{R}\left(e_{\alpha}+e_{-\alpha}\right),
$$

$A=H_{\mathbb{R}}$, we obtain a Cartan decomposition for $L_{s}$. As it follows from the results of $[4,8]$, the function $f_{n}$ on $I_{1}$ in this situation is also a perfect Morse function. It seems that the case of a general semisimple Lie algebra over $\mathbb{R}$ can be considered with the help of the technique developed in [1].

\section{CONCLUDING REMARKS}

In the present paper we have described a complete phase portrait of a generalized Toda flow on a semisimple noncompact Lie algebra over $\mathbb{R}$ and of a corresponding $Q R$-algorithm. In particular, we have shown that these dynamical systems possess the Morse-Smale property (e.g., structurally stable). We have applied the results obtained to the study of topological properties of isospectral manifolds $I_{k}$ that are natural generalizations of the isospectral manifold of tridiagonal symmetric matrices. The study of these manifolds is a necessary preliminary step towards a solution of the inverse spectral problem for the situation considered.

\section{REFERENCES}

1. M. F. Atiyah and R. Bott, Yang-Mills equations over Riemann surfaces, Philos. Trans. Roy. Soc. London Ser. A 308 (1982), 523-615.

2. R. Bott, An application of the Morse theory to the topology of compact Lie groups, Bull. Soc. Math. France 84 (1956), 251-282.

3. _ Representation Theory of Lie groups, Cambridge University Press, Cambridge, 1979, pp. 65-90.

4. M. W. Davis, Some aspherical manifolds Duke Math. J. 55 (1987), 105-139.

5. L. E. Faybusovich, $Q R$-type factorizations, the Yang-Baxter equation and an eigenvalue problem of control theory, Linear Algebra Appl. (1989), 122-124, 943-971.

6. __, QR-algorithm and generalized Toda flows, Ukrain. Mat. Zh. 41 (1989), 944-952.

7. __ Generalized Toda flows, the Riccati equations on Grassmannian and the QR-algorithm, Funct. Anal. Appl. 21 (1987), 166-168. 
8. D. Fried, The cohomology of an isospectral flow, Proc. Amer. Math. Soc. 98 (1986), 363368.

9. I. M. Gelfand and V. V. Serganova, Combinatorial geometrics and torus strata on homogeneous compact manifolds, Uspekhi Mat. Nauk 42 (1987), 107-134.

10. R. Goodman and N. R. Wallach, Classical and quantum mechanical systems of Toda lattice type II, Comm. Math. Phys. 94 (1984), 177-217.

11. S. Helgason, Differential geometry, Lie groups and symmetric spaces, Academic Press, New York, 1978.

12. R. Hermann, Toda lattices, cosymplectic manifolds, kinds, Part B, Interdisciplinary Math., vol. 18, Math. Sci. Press, Brookline, MA, 1978.

13. B. Kostant, The solution to a generalized Toda lattice and representation theory, Adv. in Math. 34 (1979), 195-338.

14. J. Moser, Three integrable hamiltonian systems connected with isospectral deformations, Adv. in Math. 16 (1975), 197-220.

15. M. Shayman and F. De Mari, Hessenberg varieties and generalized Eulerian numbers for semisimple Lie groups: The classical cases, preprint 1988.

16. W. Symes, The $Q R$-algorithm and scattering for the finite nonperiodic Toda lattice, Phys. D 4 (1982), 278-280.

Department of Mathematics, University of Notre Dame, P.O. Box 398, Notre Dame, INDIANA 46556

E-mail address: leonid@cartan.math.nd.edu 